\title{
Magnetic Resonance Imaging and Computed Tomography Findings in Pediatric Giant Cell Glioblastoma
}

\author{
L. Zipp · K. M. Schwartz · E. Hewer ' Y. Yu • \\ C. Stippich · J. M. Slopis
}

Received: 10 November 2011 / Accepted: 3 January 2012 / Published online: 28 January 2012

(C) Springer-Verlag 2012

\section{Introduction}

Giant cell glioblastoma (GCG) is considered to be a subtype of glioblastoma multiforme (GBM). This malignant tumor of glial origin is a grade IV tumor according to the current classification of the World Health Organization (WHO, [1]) and accounts for approximately $5 \%$ of GBMs and $0.8 \%$ of all brain tumors [2]. In pediatric patients supratentorial tumors are less common than infratentorial tumors with a ratio of approximately 1:2. The GBMs account for $7-9 \%$ of all intracranial tumors and $0.6-7.9 \%$ of all GBMs occur during childhood [3]. These statistics demonstrate that GCG is an exceedingly rare tumor mainly described in isolated case reports or series with a predilection for younger patients $[2,4]$. The GCGs arise from white matter, as do the common GBMs and have a tendency to involve the fron-

L. Zipp, MS $(\bowtie) \cdot$ J. M. Slopis, MD

Department of Pediatrics, MD Anderson Cancer Center,

1515 Holcombe Blvd., 77030 Houston, Texas, USA

L. Zipp, MS · C. Stippich, MD

Department of Neuroradiology, University Hospital Basel, Petersgraben 4, 4031 Basel, Switzerland

e-mail: lisa.zipp@web.de

K. M. Schwartz, MD

Department of Radiology, Mayo Clinic, 200 First St SW, 55905 Rochester, Minnesota, USA

E. Hewer, MD

Department of Neuropathology, University Hospital Basel,

Petersgraben 4, 4031 Basel, Switzerland

Y. Yu, $\mathrm{PhD}$

Department of Radiation Oncology, Kimmel Cancer Center, Thomas Jefferson University, 111 S. 11th Street,

19107 Philadelphia, Pennsylvania, USA tal and temporal lobes $[2,5]$. The higher predilection for the cerebral hemispheres than a typical GBM, probably in addition to histological variations, may be responsible for the better prognosis as the location means that gross total resection is more frequent. The tumor is macroscopically well-circumscribed and histologically shows numerous bizarre multinucleated giant cells with an abundant reticulin network and a high frequency of p53 mutations [6, 7]. In general terms GBM has a poor prognosis with a mean survival time ranging from 12-15 months in adults [8]. In pediatric patients with high grade astrocytoma the outcome is slightly better but still remains poor with survival times ranging from 15-42 months $[9,10]$. In standard GBM treatment protocols tumor resection is followed by irradiation and DNA alkylating chemotherapy. Adding temozolomide as a chemotherapy drug significantly improved outcome in newly diagnosed GBM $[11,12]$ and the median survival time improved from 12.1 to 14.6 months and the 2-year survival rate reached $25.6 \%$ compared to $10.4 \%$ previously when adding temozolomide to standard GBM treatment protocols $[13,14]$. Other factors associated with decreased survival in GBM include but are not limited to larger tumor size [15], higher age [16] as well as bilateral location [17]. The GCG is associated with longer survival times compared to GBM, ranging from 15 months up to 17 years in adults [5, $18]$ and ranging from 14 months up to 12 years in children $[4,19]$.One case report also reported a long-term survival with a late transformation to gliosarcoma [6].

\section{Case Report}

This article reports the case of an 8-year-old boy presenting at the university hospital with vomiting and a seizure lasting approximately $2 \mathrm{~min}$. A second generalized seizure was 
observed later the same day. Magnetic resonance imaging (MRI) was performed on a $1.5 \mathrm{~T}$ Magnetom Symphony scanner (Siemens Medical Systems, Erlangen, Germany) with the following imaging parameters: T2-weighted sequence $\mathrm{TR} / \mathrm{TE}=4920 / 104 \mathrm{~ms}, \quad \mathrm{~T} 1$-contrast-enhanced sequence $\mathrm{TR} / \mathrm{TE}=663 / 17 \mathrm{~ms}$, T1-fat-saturated contrastenhanced sequence TR/TE $=559 / 17 \mathrm{~ms}$, diffusion-weighted imaging and apparent diffusion coefficient (DWI/ADC) sequence TR/TE $=4400 / 105 \mathrm{~ms}$, slice thickness $5 \mathrm{~mm}$. As MRI contrast agent $10 \mathrm{ml}$ of dotarem (gadolinium-tetraazacyclododecanetetraacetic acid, Gd-DOTA) was adminis- tered. Computed tomography (CT) was performed using a 10-row multidetector CT scanner (Sensation 10, Siemens Medical Systems, Erlangen, Germany) with a slice thickness of $3 \mathrm{~mm}$. As CT contrast agent $45 \mathrm{ml}$ of ultravist was administered. The MRI and CT findings are demonstrated in Fig. 1. The CT image after contrast administration displayed an enhancing mass adjacent to the frontal horn of the left lateral ventricle and MRI showed a predominantly T2-hyperintense mass with central nodular enhancement and surrounding $\mathrm{T} 2$ hyperintensity.
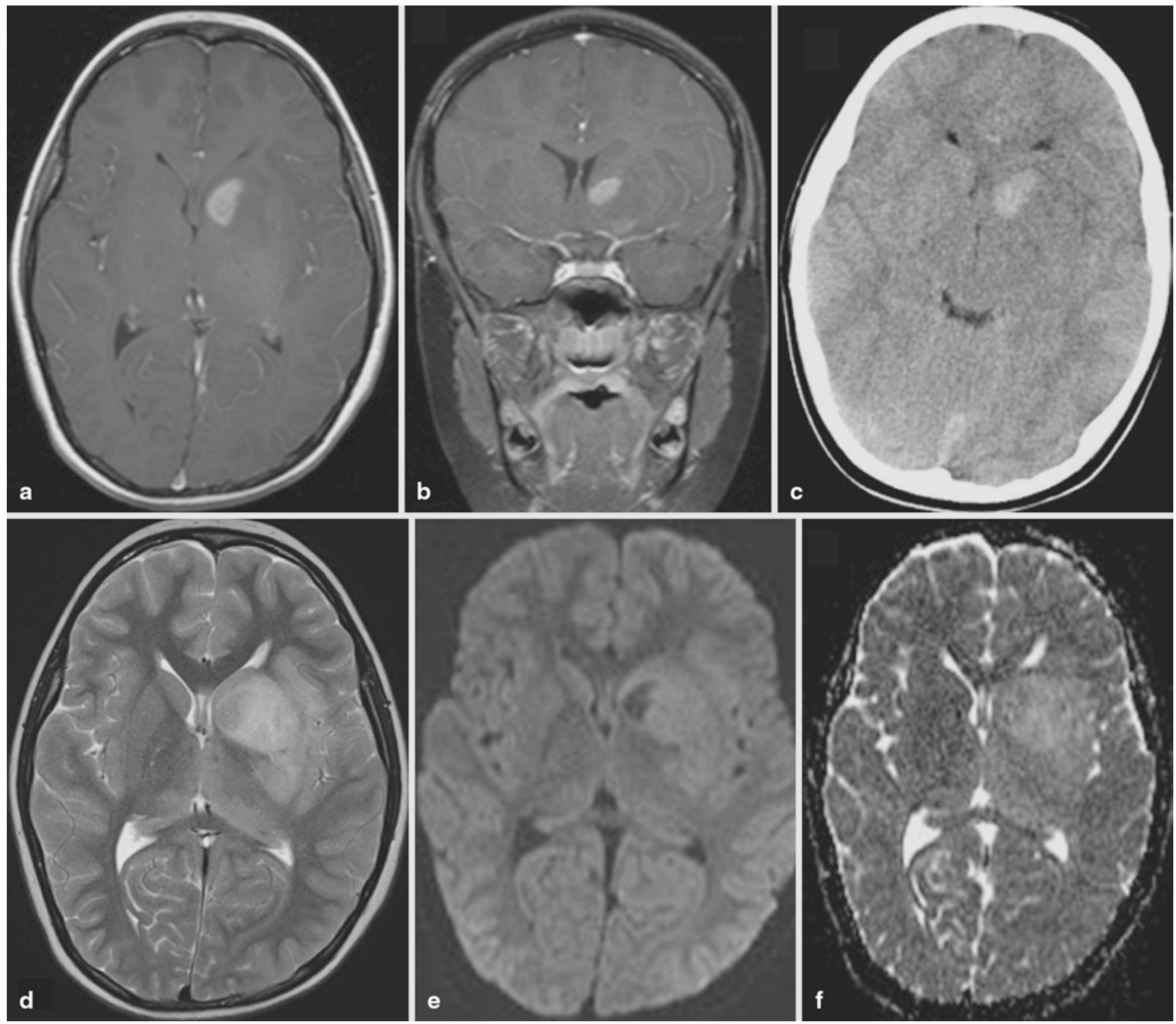

Fig. 1 An 8-year-old boy with pathologically documented giant cell glioblastoma. Contrast-enhanced axial (a) and coronal (b) T1-weighted magnetic resonance imaging (MRI) and axial postcontrast computed tomography $(\mathrm{CT}, \mathbf{c})$ images demonstrate an enhancing mass centered at the anterior limb of the left internal capsule. (d) Axial T2weighted MRI image reveals moderate surrounding T2 hyperintensity consistent with tumor and edema involving the basal ganglia and the internal and external capsules and compressing the anterior horn of the left ventricle. (e) Diffusion-weighted image (DWI) signal intensity increases in the tumor corresponding to T2 signal changes (edema and T2 shine through). The semilunar T1 contrast-enhancing part has very low DWI signals corresponding to facilitated diffusion and indicating reduced cellularity. (f) Corresponding apparent diffusion coefficient (ADC) map. The ADC value setting a region of interest of $150 \mathrm{~mm}^{2}$ within the tumor was $1168.5 \pm 1.7$ as mean value with a range between 976 (minimum value) and 1313 (maximal value) 
Fig. 2 (a) Histologically the brain biopsy showed a cellular, mitotically active glial tumor (a: Original magnification $\mathrm{x} 40$ ) and $(\mathbf{b}, \mathbf{c})$ bizarre, occasionally multinucleated giant tumor cells were also seen. (d) Immunohistochemically a pronounced nuclear p53 accumulation was present in most tumor cells. (b-d: Original magnification x200) Staining: a-c: hematoxylin and eosin; d: immunohistochemical staining for $\mathrm{p} 53$
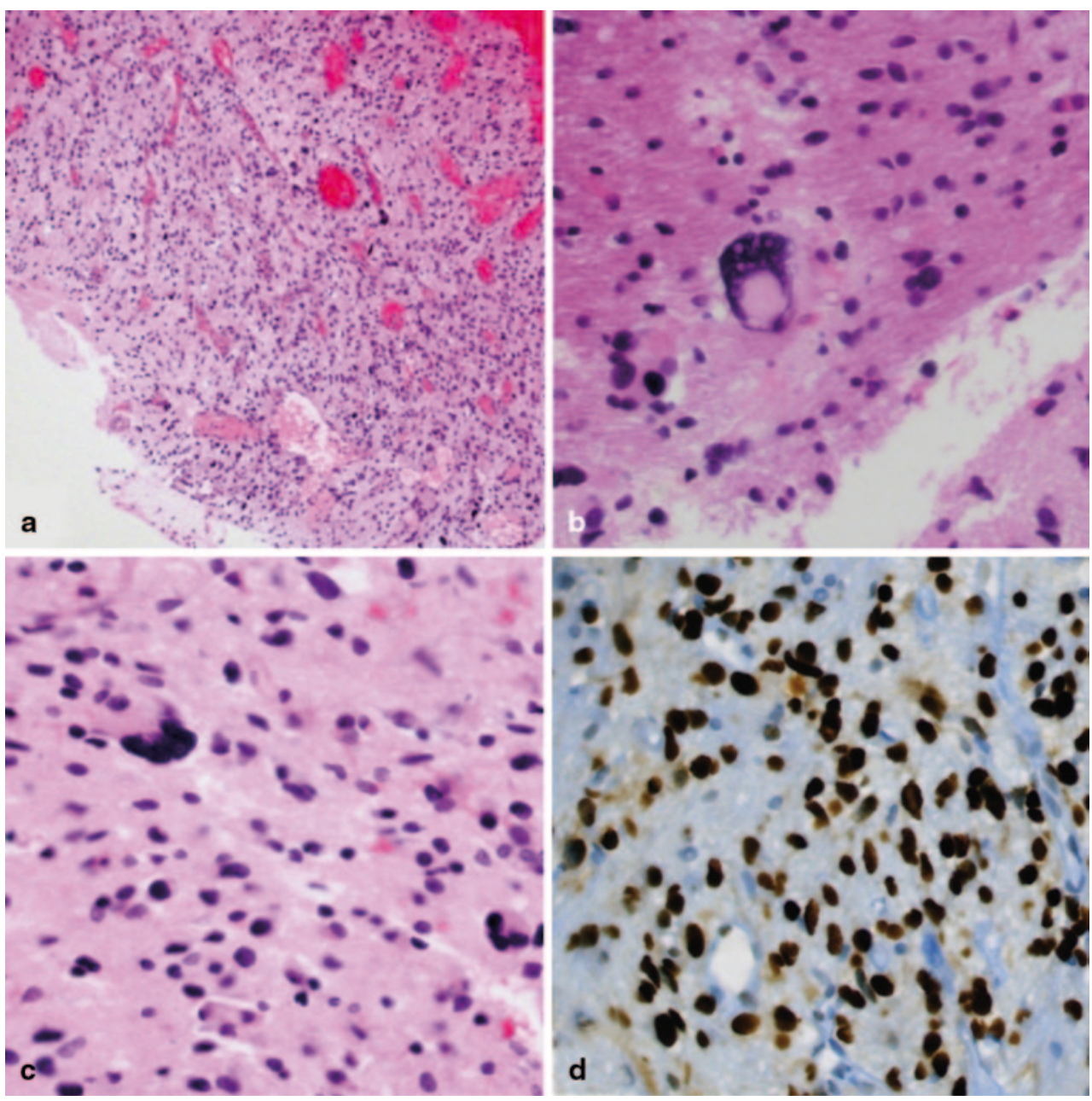

A stereotactic biopsy revealed a GCG with typical p53 accumulation in immunohistochemical staining indicating presence of TP53 mutation in the tumor as demonstrated in Fig. 2. Focal necrosis and beginning endothelial proliferation were identified, typical features to differentiate a GBM from an anaplastic WHO III glioma. Expression of glial fibrillary acidic protein (GFAP) and microtubule-associated protein 2 (MAP-2) was evident and the Ki-67 marker determined a proliferation rate of $20 \%$ (not shown). The diagnosis of GCG was confirmed by the Brain Tumor Reference Center of the German Society of Neuropathology and Neuroanatomy at the University Hospital of Bonn, Germany.

\section{Discussion}

Giant cell glioblastoma is a rare subtype of GBM and the diagnosis of GCG has significant clinical consequences. Children with a malignant glioma have a slightly better prognosis than their adult counterparts $[9,10]$. In a collection consisting of 39 pediatric patients with supratentorial and cerebellar malignant gliomas, including 1 GCG, the extent of resection was the strongest predictor of outcome [20]. In the case of a GCG where the prognosis seems to be better according to the current literature, maximum surgical resection with the combination of aggressive adjuvant radiochemotherapy (using for instance the protocols of the HIT-GBM trials [19]) may lead to better outcome. However, a previous publication could not confirm a better overall survival and event-free survival in pediatric GCGs compared to GBM [19]. The GCGs are of glial origin [21] and therefore stain positively for GFAP. Nuclear p53 accumulation was detected in the biopsy, indicating a TP53 mutation consistent with previous work on the genetic profile of GCGs with a p53 mutation frequency of more than $70 \%$ whereas epidermal growth factor receptor (EGFR) amplification was found very rarely $[22,23]$. The detected proliferation rate for the antibody against ki67 was also consistent with previous case reports determining similar or the same proliferation index of $20 \%$ as in this case [4].

The differential diagnosis based on imaging and clinical presentation is broad, including low-grade tumors, such as ganglioglioma, pleomorphic xanthoastrocytoma, and oligodendroglioma. High-grade neoplasms and infections can 
also have a similar appearance. Due to the fact that seizures and headache are very common and non-specific symptoms occur in different brain tumors, it is crucial to perform radiological scrutiny to narrow the diagnosis to improve patient management prior to treatment or biopsy.

In this case the radiological findings of an exceedingly rare primary brain tumor, a GCG, a GBM subtype probably with a better prognosis are presented. Central homogeneous enhancement is present, different than the heterogeneous peripheral enhancement often seen in classical GBMs. The contrast-enhancing part revealed a low signal on DWI indicating reduced cellularity.

Larger studies evaluating imaging findings in GCGs are needed to determine if these imaging characteristics are typical of GCGs and can help distinguish GBMs from GCGs. Although rare preoperative consideration of this entity should be included when radiologically and clinically appropriate which in turn might impact different patient care in pediatric GCGs and GBMs. In a retrospective analysis significantly more pediatric patients with GCG were treated according to the HIT-GBM-D protocol, a very intensive chemotherapy regimen, compared to pediatric GBM patients [19]. This study described the use of different neurooncological treatment protocols for GBM and GCG. The outcome of the GCG patients treated with the more intensive chemotherapy regimen was not analyzed. Hence further investigations are necessary in terms of an outcome benefit in GCG before a more intensive chemotherapy regimen can be justified. Nevertheless it is crucial to consider that histological confirmation of the exact diagnosis is required before appropriate treatment decisions can be made.

\section{Conclusions}

Preoperative consideration of a GCG should be included in pediatric patients when radiologically and clinically appropriate as well as histologically proven which in turn might influence future therapy approaches towards a more intensive chemotherapy regimen. To the best of our knowledge this is the first manuscript demonstrating MRI and CT findings in a patient with GCG.

\footnotetext{
Acknowledgment We wish to thank Prof. T. Pietsch of the Brain Tumor Reference Center of the German Society of Neuropathology and Neuroanatomy at the University Hospital of Bonn, Germany for confirmation of the histological diagnosis.
}

Conflict of Interest The authors declare that they have no conflict of interest.

\section{References}

1. Kleihues P, Louis DN, Scheithauer BW, Rorke LB, Reifenberger $\mathrm{G}$, Burger PC, et al. The WHO classification of tumors of the nervous system. J Neuropathol Exp Neurol. 2002;61(3):215-25; discussion 226-9.

2. De Prada I, Cordobes F, Azorin D, Contra T, Colmenero I, GlezMediero I. Pediatric giant cell glioblastoma: a case report and review of the literature. Childs Nerv Syst. 2006;22(3):285-9.

3. Artico M, Cervoni L, Celli P, Salvati M, Palma L. Supratentorial glioblastoma in children: a series of 27 surgically treated cases. Childs Nerv Syst. 1993;9(1):7-9.

4. Klein R, Molenkamp G, Sorensen N, Roggendorf W. Favorable outcome of giant cell glioblastoma in a child. Report of an 11year survival period. Childs Nerv Syst. 1998;14(6):288-91.

5. Margetts JC, Kalyan-Raman UP. Giant-celled glioblastoma of brain. A clinico-pathological and radiological study of ten cases (including immunohistochemistry and ultrastructure). Cancer. 1989;63(3):524-31.

6. Deb P, Sharma MC, Chander B, Mahapatra AK, Sarkar C. Giant cell glioblastoma multiforme: report of a case with prolonged survival and transformation to gliosarcoma. Childs Nerv Syst. 2006;22(3):314-9.

7. Raco A, Bristot R, Salvati M, Delfini R. Malignant supratentorial astrocytomas of late childhood. Our experience with 25 cases. Childs Nerv Syst. 1997;13(6):341-4.

8. Mirimanoff RO, Gorlia T, Mason W, Van Den Bent MJ, Kortmann RD, Fisher B, et al. Radiotherapy and temozolomide for newly diagnosed glioblastoma: recursive partitioning analysis of the EORTC 26981/22981-NCIC CE3 phase III randomized trial. J Clin Oncol. 2006;24(16):2563-9.

9. Campbell JW, Pollack IF, Martinez AJ, Shultz B. High-grade astrocytomas in children: radiologically complete resection is associated with an excellent long-term prognosis. Neurosurgery. 1996;38(2):258-64.

10. Pollack IF, Hamilton RL, Burnham J, Holmes EJ, Finkelstein SD, Sposto R, et al. Impact of proliferation index on outcome in childhood malignant gliomas: results in a multi-institutional cohort. Neurosurgery. 2002;50(6):1238-44; discussion 1244-5.

11. Malhi H, Gores GJ. TRAIL resistance results in cancer progression: a TRAIL to perdition? Oncogene. 2006;25(56):7333-5.

12. Cimini A, Ippoliti R. Innovative therapies against human glioblastoma multiforme. ISRN Oncol. 2011;2011:787490.

13. Stupp R, Mason WP, van den Bent MJ, Weller M, Fisher $\mathrm{B}$, Taphoorn MJ, et al. Radiotherapy plus concomitant and adjuvant temozolomide for glioblastoma. $N$ Engl $\mathrm{J}$ Med. 2005;352(10):987-96.

14. Hegi ME, Diserens AC, Gorlia T, Hamou MF, de Tribolet N, Weller M, et al. MGMT gene silencing and benefit from temozolomide in glioblastoma. N Engl J Med. 2005;352(10):997-1003.

15. Chaichana KL, Chaichana KK, Olivi A, Weingart JD, Bennett R, Brem $\mathrm{H}$, et al. Surgical outcomes for older patients with glioblastoma multiforme: preoperative factors associated with decreased survival. J Neurosurg. 2011;114(3):587-94.

16. Filippini G, Falcone C, Boiardi A, Broggi G, Bruzzone MG, Caldiroli D, et al. Prognostic factors for survival in 676 consecutive patients with newly diagnosed primary glioblastoma. Neuro Oncol. 2008;10(1):79-87.

17. Bauchet L, Mathieu-Daudé H, Fabbro-Peray P, Rigau V, Fabbro $\mathrm{M}$, Chinot $\mathrm{O}$, et al. Oncological patterns of care and outcome for 952 patients with newly diagnosed glioblastoma in 2004. Neuro Oncol. 2010;12(7):725-35.

18. Sabel M, Reifenberger J, Weber RG, Reifenberger G, Schmitt HP. Long-term survival of a patient with giant cell glioblastoma. Case report. J Neurosurg. 2001;94(4):605-19. 
19. Karremann M, Butenhoff S, Rausche U, Pietsch T, Wolff JE, Kramm CM. Pediatric giant cell glioblastoma: new insights into a rare tumor entity. Neuro Oncol. 2009;11(3):323-9.

20. Bucci MK, Maity A, Janss AJ, Belasco JB, Fisher MJ, Tochner ZA, et al. Near complete surgical resection predicts a favourable outcome in pediatric patients with nonbrainstem, malignant gliomas: results from a single center in the magnetic resonance imaging era. Cancer. 2004;101(4):817-24.

21. Kawano H, Kubota T, Sato K, Goya T, Arikawa S, Wakisaka S. Immunohistochemical study of giant cell in glioblastoma. Clin Neuropathol. 1995;14(2):118-22.
22. Martinez-Diaz H, Kleinschmidt-DeMasters BK, Powell SZ, Yachnis AT. Giant cell glioblastoma and pleomorphic xanthoastrocytoma show different immunohistochemical profiles for neuronal antigens and p53 but share reactivity for class III beta-tubulin. Arch Pathol Lab Med. 2003;127(9):1187-91.

23. Peraud A, Watanabe K, Schwechheimer K, Yonekawa Y, Kleihues P, Ohgaki H. Genetic profile of the giant cell glioblastoma. Lab Invest. 1999;79(2):123-9. 